\title{
Modulatory Interactions between the Default Mode Network and Task Positive Networks in Resting-State
}

The two major brain networks, i.e. the default mode network (DMN) and the task positive network, typically reveal negative and variable connectivity in resting-state. In the present study, we examined whether the connectivity between the DMN and different components of the task positive network were modulated by other brain regions by using physiophysiological interaction (PPI) on resting-state functional magnetic resonance imaging data. Spatial independent component analysis was first conducted to identify components that represented networks of interest, including the anterior and posterior DMNs, salience, dorsal attention, left and right executive networks. PPI analysis was conducted between pairs of these networks to identify networks or regions that showed modulatory interactions with the two networks. Both network-wise and voxel-wise analyses revealed reciprocal positive modulatory interactions between the DMN, salience, and executive networks. Together with the anatomical properties of the salience network regions, the results suggest that the salience network may modulate the relationship between the DMN and executive networks. In addition, voxel-wise analysis demonstrated that the basal ganglia and thalamus positively interacted with the salience network and the dorsal attention network, and negatively interacted with the salience network and the DMN. The results demonstrated complex modulatory interactions among the DMNs and task positive networks in resting-state, and suggested that communications between these networks may be modulated by some critical brain structures such as the salience network, basal ganglia, and thalamus. 
1 Modulatory Interactions between the Default Mode Network and Task Positive Networks in

2 Resting-State

3 Xin Di, Bharat B. Biswal*

4 Department of Biomedical Engineering, New Jersey Institute of Technology, Newark, NJ, 07102, 5 USA.

6 *Corresponding author:

7 Bharat B. Biswal, $\mathrm{PhD}$

8607 Fenster Hall, University Height

9 Newark, NJ, 07102, USA

10 bbiswal@yahoo.com 


\section{Introduction}

12 The human brain is intrinsically organized as different networks as generally revealed by resting-

13 state functional magnetic resonance imaging (fMRI) (Beckmann et al., 2005; Golland et al.,

14 2008; Yeo et al., 2011). Brain regions within a network generally convey relatively higher

15 connectivity than regions from different networks (Biswal et al., 1995; Cordes et al., 2000;

16 Greicius et al., 2003), thus constituting modular organizations of brain functions (Salvador et al.,

17 2005; Meunier et al., 2009; Doucet et al., 2011). On the other hand, brain regions that belonged

18 to different networks generally have weaker connectivity, however, between network

19 communications are considered to be critical to support complex brain functions which need to

20 integrate resources from different brain systems (Bullmore and Sporns, 2012; Cole et al., 2013).

21 There are two major systems in the brain, the task positive network shows consistent

22 activations across different tasks (Shulman et al., 1997a), while the default mode network (DMN)

23 shows consistent deactivations (Shulman et al., 1997b). These two systems reveal moment to

24 moment anticorrelation even when the subject isn't performing explicit tasks (Fox et al., 2005).

25 The negative correlation between the DMN and the task positive network becomes stronger after

26 adolescence (Barber et al., 2013; Chai et al., 2014), and may serve as a suppression mechanism

27 that inhibits unwanted thoughts, thus making behavior responses more reliable (Kelly et al.,

28 2008; Spreng et al., 2010; Anticevic et al., 2012; Wen et al., 2013). Although the original study

29 of anticorrelation has been questioned because of global regression in data processing (Murphy et

30 al., 2009), further studies have shown that the negative correlation between the DMN and the task

31 positive network is still present without global regression (Fox et al., 2009; Chai et al., 2012), and

32 thought to be of neuronal origins (Keller et al., 2013). However, the controversies of negative

33 correlation may partially due to the fact that the connectivity between the DMN and the task

34 positive network are highly variable (Chang and Glover, 2010; Kang et al., 2011). 
The negative connectivity between the task positive network and DMN has been shown to

36 be modulated or mediated by other networks, which may provide hints on the variability of the

37 negative correlation. Sridharan and colleagues showed that the salience network (Seeley et al.,

38 2007) activated the executive network which is part of the task positive network, and deactivated

39 the DMN during both task performing conditions and resting-state (Sridharan, Levitin, and

40 Menon, 2008). In addition, Spreng and colleagues suggested that the relationship between the

$41 \mathrm{DMN}$ and the dorsal attention network was mediated by regions of the frontoparietal control

42 network (Spreng et al., 2013). Thus, the task positive network could be further divided into

43 different sub-networks such as the salience network, dorsal attention network, and (left and right)

44 executive networks, and these networks may convey complex interactions with the DMN. In the

45 present study, we aimed to investigate whether the relationship between two networks was

46 modulated by other networks (or regions) by using physiophysiological interaction (PPI) (Friston

47 et al., 1997; Di and Biswal, 2013a), which might provide a novel avenue to characterize complex

48 relationships among these networks.

49 Specifically, we sought to systematically investigate the modulatory interaction between

50 the DMN and task positive networks using PPI analysis on resting-state fMRI data. Spatial

51 independent component analysis (ICA) was first performed to identify the networks of interest,

52 including the anterior and posterior DMNs, salience, dorsal attention, left executive, and right

53 executive networks. PPI analysis was then performed between each two of the networks using

54 both network-wise and voxel-wise analyses. This between-network PPI analysis was used to

55 identify networks or regions that modulate the dynamic relationship between the two predefined

56 networks. Based on notion that the salience network played an important role in switching of

57 large scale brain networks (Sridharan, Levitin, and Menon, 2008; Menon and Uddin, 2010), we

58 predicted that the salience network might show interaction effects with the DMN and executive

59 networks. 


\section{Methods}

\section{Subjects}

62 The resting-state fMRI data was derived from the Beijing_Zang dataset of the 1000 functional 63 connectomes project (http://fcon_1000.projects.nitrc.org/) (Biswal et al., 2010). This dataset

64 originally contained 198 subjects. The first 64 subjects without large head motions were included 65 in the current analysis (40 female/ 24 male). The mean age of these subjects was 21.1 years 66 (range from 18 to 26 years of age). This study involves analyzing public available dataset, which

67 doesn't need IRB approval. Further, we didn't use any patient identification features in this study.

\section{Scanning parameters}

69 The MRI data were acquired using a SIEMENS Trio 3-Tesla scanner from Beijing Normal

70 University. 230 resting-state functional data were acquired for each subject using TR of $2 \mathrm{~s}$. The

71 resolution of the fMRI images was $3.125 \times 3.125 \times 3 \mathrm{~mm}^{3}$ with 64 x 64 x 36 voxels. T1-

72 weighted three-dimensional magnetization-prepared rapid gradient echo (MP-RAGE) images

73 were acquired for all the subject using the following parameters: 128 slices, $\mathrm{TR}=2530 \mathrm{~ms}, \mathrm{TE}=$

$743.39 \mathrm{~ms}$, slice thickness $=1.33 \mathrm{~mm}$, flip angle $=7^{\circ}$, inversion time $=1100 \mathrm{~ms}, \mathrm{FOV}=256 \times 256$

$75 \mathrm{~mm}^{2}$.

\section{Functional MRI data analysis}

\section{Preprocessing}

78 The fMRI image preprocessing and analysis were conducted using SPM8 package

79 (http://www.fil.ion.ucl.ac.uk/spm/) under MATLAB 7.6 environment

80 (http://www.mathworks.com). For each subject, the first two functional images were discarded,

81 resulting in 228 images for each subject. Firstly, the functional images were motion corrected

82 using the realign function. The head motion estimates in any of the three translational or three

83 rotational directions for all the subjects were less than $2 \mathrm{~mm}$ or $2^{\circ}$. Next, the functional images 
84 were linearly coregistrated to the subject's own high resolution anatomical image using the

85 coregister function. Next, subject's anatomical images were normalized to the T1 template

86 provided by SPM package in MNI space (Montreal Neurological Institute). Then, the

87 normalization parameters were used to normalize all the functional images into MNI space, and

88 the functional images were resampled into $3 \times 3 \times 3 \mathrm{~mm}^{3}$ voxels. Finally, all the functional

89 images were smoothed using a Gaussian kernel with $8 \mathrm{~mm}$ full width at half maximum (FWHM).

90 Spatial ICA

91 Spatial ICA was conducted to define networks for the PPI analysis using Group ICA of fMRI

92 Toolbox (GIFT) (http://icatb.sourceforge.net/) (Calhoun et al., 2001). Twenty components were

93 extracted. Among the 20 ICA maps (see supplementary Figure S1), we identified the DMN and

94 task positive network components by visually comparing the IC maps with previous studies

95 (Biswal et al., 2010; Cole, Smith, and Beckmann, 2010). Two components were identified as

96 DMN, with one more anteriorly localized (Figure 1A) and the other more posteriorly localized

97 (Figure 1B). We also identified four components that represented different task positive

98 networks, i.e. the salience, dorsal attention, left executive, and right executive networks (Figure

99 1C through 1F). Time series associated with these six components were obtained for each subject

100 for following PPI analysis. To aid interpretations of the PPI results, simple correlations among

101 the six networks were calculated for each subject. The correlation values were transformed into

102 Fisher's z, and statistical significances were tested across subjects using one sample t-test.

103 [Insert Figure 1 here]

\section{PPI analysis}

105 Physiophysiological interaction analysis, along with its variant psychophysiological interaction,

106 were first proposed by Friston and colleagues to characterize modulated connectivity by another

107 region or a psychological manipulation (Friston et al., 1997). The present analysis focused on the 
108 modulation of connectivity by other regions or networks. Specifically, time series of two

109 networks were used to define an interaction model using a linear regression framework.

$$
y=\beta_{N 1} \cdot x_{N 1}+\beta_{N 2} \cdot x_{N 2}+\beta_{P P I} \cdot x_{N 1} \cdot x_{N 2}+\varepsilon
$$

$$
x_{N 1} \quad x_{N 2}
$$

110 Where and represented the time series of two networks. Critically, we were interested in

111 whether the interaction term of the two time series was correlated with the time series of a given

112 voxel or region ${ }^{y}$ (the effect of ${ }^{\beta_{P P I}}$ ). A positive interaction effect implies that the connectivity

113 between the resultant region and one of the network is positively modulated by the other network.

114 While a negative interaction effect implies that the connectivity between the resultant region and

115 one of the network is negatively modulated by the other network. It should be noted that the PPI

116 analysis is different from partial correlation analysis, which simply examines a linear relationship

117 between two regions by controlling the activity of a third region (Marrelec et al., 2006). A partial

$$
\beta_{N 1} \quad \beta_{N 2}
$$

118 correlation is similar to the effects of and in the current model where the activity of

$119 x_{N 2}^{x_{N 1}}$ or is controlled, respectively, which cannot directly examine the interaction of the two

120 variables.

121 In practice, the time series of the two networks were deconvolved with a hemodynamic

122 response function (hrf), so that the PPI term was calculated in the neuronal level but not

123 hemodynamic level (Gitelman et al., 2003). The deconvolution procedure can in principle

124 minimize noises when calculating PPI terms (Gitelman et al., 2003), and has been shown to

125 provide better statistical results in previous empirical analysis (Di and Biswal, 2013a). 
127 Six rigid-body motion parameters, the first principle component time series of white matter

128 (WM) signal, and the first principle component time series of cerebrospinal fluid (CSF) signal

129 were regressed out from the original time series by using linear regression model. The subject

130 specific WM and CSF masks were derived from their own segmented WM and CSF images, with

131 a threshold of 0.99 to make sure that GM voxels were excluded from the masks. Next, a high-

132 pass filter of $0.01 \mathrm{~Hz}$ was applied on the time series to minimize low frequency scanner drift.

133 The preprocessed time series of two networks were first deconvolved with the hrf using a simple

134 empirical Bayes procedure, so that the resulting time course represented an approximation to

135 neural activity (Gitelman et al., 2003). Next, the two neural time series were detrended and point

136 multiplied, so that the resulting time series represented the interaction of neural activity between

137 two networks. And lastly, the interaction time series was convolved with the hrf, resulting in an

138 interaction variable in BOLD level. The PPI terms were calculated for each pair of the six

139 networks.

140 Network-wise PPI analysis was first conducted to directly examine the relationships

141 among networks, which is similar to von Kriegstein and Giraud (von Kriegstein and Giraud,

142 2006). In the network-wise analysis, the dependent variable was the time series of a network

143 rather than the time series of every voxel in the brain. In the PPI linear regression model, the

144 main effects of the two networks, and the PPI effects between them were added as independent

145 variables along with a constant regressor. After model estimation, cross-subject one-sample t-

146 tests were performed on the beta values of PPI effects. The critical $p$ value was set as $p<0.05$

147 after Bonferroni correction (corresponding to a raw $\mathrm{p}$ value of $8.33 \times 10^{-4}$ after correcting for

148 totally 60 comparisons).

149 In addition, voxel-wise PPI analysis was also performed to identify regions across the

150 whole brain that were associated with a PPI effect. PPI terms were calculated for each pair of the 
151 six networks, resulting in 15 PPI effects. Then separate PPI models were built for each subject

152 using the general linear model (GLM) framework. The GLM model contained two regressors

153 representing the main effects of two networks' time series, one regressor representing the PPI

154 effect, two regressors representing WM and CSF signals, and six regressors representing head

155 motion effects. An implicit high pass filter of 1/100 Hz was used. For each PPI effect, a 2nd-

156 level one sample t-test was conducted to make group-level inference. Simple t contrast of 1 or -1

157 was defined to reveal positive or negative PPI effects, respectively. The resulting clusters were

158 first height thresholded at $\mathrm{p}<0.001$, and cluster-level false discovery rate (FDR) corrected at $\mathrm{p}<$

1590.0033 based on random field theory (Chumbley and Friston, 2009). The cluster-level p value

160 was chosen to take into account the totally 15 PPI effects. The resulting clusters were labeled

161 according to their peak coordinates using Talairach Daemon (Lancaster et al., 2000), after taking

162 into account the discrepancies between MNI space and Talairach space (Lancaster et al., 2007).

\section{Results}

\section{Simple correlations among networks}

165 The mean correlations among the six networks are listed in Table 1. There was a positive

166 correlation between the anterior and the posterior DMNs $\left(M_{\text {Fisher's } z}=0.359\right)$. However, the

167 correlations among the four task positive networks were mixed. The salience network revealed a

168 positive correlation with the dorsal attention network $\left(M_{\text {Fisher's } z}=0.333\right)$, but a negative

169 correlation with the right executive network $\left(M_{\text {Fisher's } z}=-0.142\right)$. There was a positive correlation

170 between the left and right executive networks $\left(M_{\text {Fisher's } z}=0.427\right)$. The correlations between DMN

171 components and task positive components were also mixed. The anterior DMN showed negative

172 correlations with the salience network $\left(M_{\text {Fisher's } z}=-0.299\right)$ and the dorsal attention network

$173\left(M_{\text {Fisher's } z}=-0.530\right)$, while positive correlations with the left executive network $\left(M_{\text {Fisher's } z} 0.184\right)$

174 and the right executive network $\left(M_{\text {Fisher's } z}=0.247\right)$. Similarly, the posterior DMN revealed a 
175 negative correlation with the salience network $\left(M_{F i s h e r ' s z}=-0.251\right)$, while positive correlations

176 with the left executive network $\left(M_{\text {Fisher's } z}=0.320\right)$ and the right executive network $\left(M_{\text {Fisher's } z}=\right.$ 177 0.188).

178

[Insert Table 1 here]

179 Network-wise PPI analysis

180 Significant network-wise modulatory interactions are illustrated in Figure 2 (see also

181 supplementary Table S1 for a full list of statistics). First, positive modulatory interactions were

182 observed among the DMNs, the salience network, and the executive networks. Positive

183 modulatory interactions were observed among the anterior DMN, salience, and right executive

184 networks in all of the three ways. The time series of anterior DMN were correlated with the

185 interactions of the salience and right executive networks $\left(M_{\text {beta }}=0.060 ; t=4.77, p=1.14 \times 10^{-5}\right)$.

186 The time series of salience network were correlated with the interactions of the anterior DMN

187 and right executive network $\left(M_{\text {beta }}=0.054 ; t=4.09, p=1.25 \times 10^{-4}\right)$. And the time series of the

188 right executive network were correlated with the interactions of the anterior DMN and salience

189 network $\left(M_{\text {beta }}=0.109 ; t=8.27, p=1.19 \times 10^{-11}\right)$. The left executive time series were also

190 correlated with the interactions of the anterior DMN and salience network $\left(M_{\text {beta }}=0.048 ; t=\right.$

$\left.1913.67, p=4.98 \times 10^{-4}\right)$. In addition, the time series of the right executive network were correlated

192 with the interactions of the posterior DMN and salience network $\left(M_{\text {beta }}=0.045 ; t=3.81, p=\right.$

$\left.1933.17 \times 10^{-4}\right)$. Second. a negative modulatory interaction was also observed among the anterior

194 DMN, posterior DMN and right executive network. The time series of the anterior DMN were

195 negatively correlated with the interactions of the posterior DMN and right executive network

$196\left(M_{\text {beta }}=-0.039 ; t=-0.404, p=1.48 \times 10^{-4}\right)$. Lastly, positive modulatory interactions were also

197 observed among task positive networks. The left executive network time series were correlated

198 with the interactions of the salience and right executive network $\left(M_{\text {beta }}=0.046 ; t=4.01, p=\right.$

$1991.66 \times 10^{-4}$ ), and the right executive network times series were correlated with the interactions of 
200 the salience and left executive network $\left(M_{\text {beta }}=0.053 ; t=3.94, p=2.06 \times 10^{-4}\right)$. The right

201 executive network time series were also correlated with the interaction effects of the dorsal

202 attention and left executive networks $\left(M_{\text {beta }}=0.058 ; t=4.31, p=5.91 \times 10^{-5}\right)$.

203

[Insert Figure 2 here]

\section{Voxel-wise PPI analysis}

205 The voxel-wise PPI results of the anterior DMN with the four task positive networks are

206 illustrated in Figure 3. A full list of regions that showed significant PPI effects in all the fifteen 207 voxel-wise analyses can also been found in supplementary Table S2. The regions that revealed 208 positive modulatory interactions with the anterior DMN and salience network mainly resembled a 209 typical task positive network (Figure 3A). These regions included the bilateral dorsolateral

210 prefrontal cortex (mainly the middle and superior frontal gyrus, BA 9 and 10), bilateral parietal

211 lobule (mainly the precuneus and inferior parietal lobule, BA 7 and 40), and left middle temporal 212 gyrus (BA 37). Additionally, a small cluster in the posterior cingulate (BA 29) also revealed

213 positive modulatory interactions. In contrast, several regions showed negative modulatory

214 interactions, including the middle portion of cingulate gyrus (BA 24), bilateral putamen, and right

215 insula (BA 13). For the modulatory interactions of the anterior DMN and dorsal attention

216 network, positive effects were observed in the bilateral dorsolateral prefrontal cortex (mainly the

217 middle and superior frontal gyrus, BA 9, and 47), and bilateral parietal lobule (mainly the inferior

218 parietal lobule and supramarginal gyrus, BA 40) (Figure 3B). No negative effects were observed.

219 Only one region located in the right inferior parietal lobule (BA 40) revealed negative modulatory

220 interactions with the anterior DMN and left executive network (Figure 3C). No positive effects

221 were observed. For the modulatory interactions of the anterior DMN and right executive network

222 (Figure 3D), positive effects were observed in the bilateral insula (BA 13), middle portion of

223 cingulate gyrus (BA 24), right inferior parietal lobule (BA 40), and right middle frontal gyrus 
224 (BA 10). The bilateral insula and cingulate gyrus resembled the typical salience network.

225 Negative effects were observed in the right middle frontal gyrus (BA 6).

226

227

228

229

230

231

232

233

234

235

236

237

238

239

240

241

242

243

244

245

246

247

248

[Insert Figure 3 here]

The voxel-wise PPI results of the posterior DMN with the four task positive networks are shown in Figure 4. Only positive effects were observed in the modulatory interactions of the posterior DMN and salience network, which were localized in the anterior portion of cingulate gyrus (BA 32), posterior portions of cingulate gyrus (BA 31), and left inferior parietal lobule (BA 40) (Figure 4A). For the modulatory interactions of the posterior DMN and dorsal attention network, only positive effects were observed, which were localized in the right middle occipital gyrus (BA 19), left inferior and middle frontal gyrus (BA 44/47), right cerebellum, and left supramarginal gyrus (BA 40) (Figure 4 B). No significant modulatory interactions were found between the posterior DMN and left or right executive networks.

[Insert Figure 4 here]

The PPI results of networks within the DMN and within task positive networks are shown in Figure 5. Only negative effects were found for the modulatory interactions between the anterior and posterior DMNs, which were localized in the superior frontal gyrus (BA 6), left middle occipital gyrus (BA 19), and right precuneus (BA 7). For the modulatory interactions of the salience network and dorsal attention network (Figure 5B), positive effects were observed in the medial frontal gyrus (BA 6), subcortical nuclei including right thalamus and left claustrum, and right postcentral gyrus (BA 2). Negative effects were observed in the left inferior frontal gyrus (BA 9). For the modulatory interactions of the salience network and left executive network (Figure 5C), positive PPI effects were observed in the medial frontal gyrus (BA 8), left superior temporal gyrus (BA 39), and left middle frontal gyrus (BA 6). No negative effects were observed. For the modulatory interactions of the salience network and right executive network (Figure 5D), positive effects were observed in the superior frontal gyrus (BA 8), right inferior 
249 frontal gyrus (BA 47), right superior temporal gyrus (BA 39), and right precentral gyrus (BA 9).

250 No negative PPI effects were observed. For the modulatory interactions of the dorsal attention

251 network and left executive network (Figure 5E), positive effects were observed in the left inferior

252 parietal lobule (BA 40) and left middle frontal gyrus (BA 6). No negative effects were observed.

253 Only one cluster in the right precuneus (BA 39) revealed positive modulatory interactions with

254 the dorsal attention network and right executive network (Figure 5F). Lastly, for the modulatory

255 interactions of the left and right executive networks (Figure 5G), positive PPI effects were

256 observed in the bilateral precuneus (BA 7). No negative effects were observed.

[Insert Figure 5 here]

\section{Discussion}

259 Similar to previous studies, we observed negative correlations between the DMN and some task

260 positive networks, for example between the salience network and anterior or posterior DMNs,

261 and between the dorsal attention network and anterior DMN. However, both the anterior and

262 posterior DMNs revealed small to moderate positive correlations with both the left and right

263 executive networks. These results suggested complex relationships between the DMNs and

264 different task positive networks. It should be noted that the absolute correlation values are

265 subject to preprocessing strategies and levels of noises (Fox et al., 2009; Weissenbacher et al.,

266 2009; Saad et al., 2012), so that examining the modulations of connectivity may provide

267 complementary supports of functional interactions between networks or regions. The current PPI

268 results can be summarized as follows. First, there were positive modulatory interactions among

269 the DMN, the salience network and the executive networks. Second, there were negative

270 modulatory interactions among the anterior DMN, posterior DMN, and right executive network.

271 Third, there were positive modulatory interactions among task positive networks, specifically the

272 salience network with the left and right executive networks, and the dorsal attention network with 
273 the left and right executive networks. And finally, voxel-wise analysis also revealed some

274 interesting findings, e.g. the subcortical regions such as the basal ganglia and thalamus were

275 negatively associated with the interactions of the anterior DMN and the salience network, but

276 were positively associated with the interactions of the salience network and the dorsal attention

277 network.

278 The modulatory interaction among the DMN, the salience network, and the executive

279 networks were mainly among the anterior DMN, the salience network and the right executive

280 network. These results can be observed not only in the network-wise analysis, but also in the

281 voxel-wise analysis. For example, the analysis of the anterior DMN and the salience network

282 revealed clusters that resembled the bilateral executive network (Figure 3A). The analysis of the

283 anterior DMN and right executive network revealed clusters that resembled the salience network

284 (Figure 3D). Lastly, the analysis of the salience network and the right executive network

285 revealed clusters that were part of the DMN (Figure 5D). The left executive network also

286 showed association with the interactions of the anterior DMN and the salience network in both

287 the PPI-wise and voxel-wise analyses (Figure 3A). In addition, the right executive network

288 showed interactions with the posterior DMN and the salience network in the network-wise

289 analysis. These results are consistent with our recent findings that the connectivities between the

290 DMN regions and frontoparietal regions were positively modulated by the salience network

291 activities, which used an independent subject sample to the current analysis (Di and Biswal, 292 2013b).

A significant PPI effect can be explained as a modulation of connectivity between two

294 regions by the third region, or equivalently as two regions having a nonlinear multiplicative effect

295 on the third region. Due to the nature of regression model used in PPI analysis, the role of each

296 region can only be implied in conjunction with other evidences such as brain anatomy and causal

297 influences. Among the DMN, salience, and executive networks, the salience network may play a 
critical role. Anatomically, the salience network contains a special type of neurons termed von

299 Economo neuron (Allman et al., 2010), which are spindle like bipolar neurons with thick axons.

300 These properties may enable von Economo neurons to rapidly pass information from the salience

301 network regions to other brain regions (Butti et al., 2009). In terms of causal influences, studies

302 using Granger causality analysis suggested that the salience network exerted influence to both the

303 DMN and executive networks (Sridharan, Levitin, and Menon, 2008; Liao et al., 2010;

304 Deshpande, Santhanam, and Hu, 2011; Yan and He, 2011). Taken together, a possible

305 explanation of the PPI results may be that the salience network, in addition to activating the

306 executive network and deactivating the DMN (Sridharan, Levitin, and Menon, 2008), directly

307 modulate the relationship between the executive network and DMN.

308 The modulation may reflect that saliency signals conveyed by the salience network

309 increase the communication between the executive system and internal oriented system.

310 Alternatively, because the absolute connectivity between the executive network and the DMN is

311 subject to preprocessing strategies, and these two networks are generally considered as

312 anticorrelated (Fox et al., 2005; Chai et al., 2012; Keller et al., 2013), it is also possible that the

313 modulation may reflect decreased anticorrelation between the DMN and executive networks.

314 The decreased anticorrelation might suggest an absence of modulation of top-down signals from

315 the DMN to central executive regions (Anticevic et al., 2012). In line with this notion, impaired

316 salience network functions in patients of schizophrenia is coincidentally associated with altered

317 connectivity between the executive network and DMN (Manoliu et al., 2013, 2014). The

318 modulatory model of the salience network on the executive network and DMN may provide a

319 novel avenue to understand dysfunctions of network communications in patients with

320 schizophrenia (Menon, 2011).

321 In contrast, negative modulatory interactions were observed among the anterior and

322 posterior portions of the DMN and the right executive network, which were evident in both the 
323 324 5A). The voxel-wise analysis results appear similar to our previous results using the posterior

network-wise analysis and the voxel-wise analysis of the anterior and posterior DMNs (Figure cingulate gyrus (PCC) and medial prefrontal cortex (MPFC) as seed regions (Di and Biswal, 2013a). These results together with the above discussed results suggest complex relationships between the DMN and executive network, which differently modulated by the salience network and different parts of the DMN.

In addition to the modulatory interactions between the DMN and task positive networks, we also observed modulatory interactions among different task positive networks. These interactions were mainly among the salience network and bilateral executive networks, and among the dorsal attention network and bilateral executive networks. The frontoparietal executive network is generally identified bilaterally when using seed-based correlations and cluster analysis (Dosenbach et al., 2007; Yeo et al., 2011), however, separate left and right lateralized frontopareital networks can be reliably identified when using ICA (Beckmann et al., 2005; Biswal et al., 2010). The current analysis revealed a moderate correlation between the left and right executive networks (mean Fisher's z 0.43), which was the largest correlation among task positive networks, suggesting that the left and right executive networks are highly functionally related. In addition, the modulatory interactions results suggested that the relationship between the left and right frontoparietal networks may be modulated by the salience network and the dorsal attention network. A previous study has suggested that the left and right lateralized executive networks may be associated with different cognitive functions, with the left executive network more associated with language cognition, and the right counterpart more related to action inhibition and pain perception (Smith et al., 2009). The increased connectivity between the bilateral networks may reflect the increased communication of resources from different executive systems. 
348 interactions with different networks, notably the thalamus and basal ganglia. Specifically, the

349 bilateral putamen of the basal ganglia revealed negative modulatory interactions with the anterior

350 DMN and salience network (Figure 3A), while the more medial portion of the basal ganglia

351 (mainly the globus pallidus) and the thalamus showed positive modulatory interactions with the

352 salience and dorsal attention networks (Figure 5B). The basal ganglia is functionally connected

353 to widely distributed cortical regions (Di Martino et al., 2008) possibly supported by different

354 white matter fibers (Lehéricy et al., 2004; Leh et al., 2007). Models of basal ganglia functions

355 have suggested it to be a moderator that modulate connectivity from frontal regions to posterior

356 visual areas to support task switching and attention shifting (Stephan et al., 2008; den Ouden et

357 al., 2010; van Schouwenburg et al., 2010). The current results extended these notion into resting-

358 state, suggesting a general modulating role of the basal ganglia on connectivity between brain

359 networks. The thalamus is a critical subcortical structure that involves many functions including

360 attention (O’Connor et al., 2002; Haynes, Deichmann, and Rees, 2005). It is possible that the

361 salience signal from the salience network enhance the connectivity from the thalamus to the

362 dorsal attention network to allocate attention recourses to specific stimulus (Fan et al., 2005).

363 Alternatively, the salience signal might modulate top-down connectivity from the dorsal attention

364 network to the thalamus, thus facilitating attentional gating of the salient event (McAlonan et al.,

365 2000; McAlonan, Cavanaugh, and Wurtz, 2008; Fischer and Whitney, 2012). Further studies

366 using causal models are needed to further clarify the dynamic relationships among the thalamus,

367 the salience network, and the dorsal attention network (Friston, Harrison, and Penny, 2003; Di

368 and Biswal, 2014).

369 By applying PPI technique to brain networks in resting-state, the current study

370 demonstrated modulatory interactions among different brain systems. Compared with our

371 previous study that examined PPI effects of two regions within the same network (Di and Biswal, 
372 2013a), the current results generally revealed larger spatial extent of significant effects. One

373 possibility is that the time series extracted from whole brain IC maps are less noisy than the time

374 series extracted from small spherical regions of interest. Another possibility is that the time

375 series from two regions of the same network may be highly correlated, thus the interaction is

376 highly correlated with the main effects. Alternatively, it may reflect that different brain regions

377 exhibit different characterizations of modulatory interactions. Some regions may dynamically

378 connected to different regions upon task demands, while other regions may be more likely to

379 stably connected to same regions. Charactering the spatial distributions of modulatory

380 interactions may strengthen our understandings of brain network dynamics. For example,

381 identifying regions that are more likely to show modulatory interactions may help to spotlight

382 important regions that may serve as flexible hubs that dynamically control different task specific

383 regions (Cole et al., 2013). 
384

385

386

387

388

390

391

392

393

394

395

396

397

398

399

400

401

402

403

404

405

406

\section{References}

Allman JM, Tetreault NA, Hakeem AY, Manaye KF, Semendeferi K, Erwin JM, Park S, Goubert V, Hof PR, 2010. The von Economo neurons in frontoinsular and anterior cingulate cortex in great apes and humans. Brain Struct. Funct. 214: 495-517.

Anticevic A, Cole MW, Murray JD, Corlett PR, Wang X-J, Krystal JH, 2012. The role of default network deactivation in cognition and disease. Trends Cogn. Sci. 16: 584-92.

Barber AD, Caffo BS, Pekar JJ, Mostofsky SH, 2013. Developmental changes in within- and between-network connectivity between late childhood and adulthood. Neuropsychologia 51: $156-167$.

Beckmann CF, DeLuca M, Devlin JT, Smith SM, 2005. Investigations into resting-state connectivity using independent component analysis. Philos. Trans. R. Soc. Lond. B. Biol. Sci. 360: 1001-13.

Biswal B, Yetkin FZ, Haughton VM, Hyde JS, 1995. Functional connectivity in the motor cortex of resting human brain using echo-planar MRI. Magn. Reson. Med. 34: 537-41.

Biswal BB, Mennes M, Zuo X-N, Gohel S, Kelly C, Smith SM, Beckmann CF, Adelstein JS, Buckner RL, Colcombe S, Dogonowski A-M, Ernst M, Fair D, Hampson M, Hoptman MJ, Hyde JS, Kiviniemi VJ, Kötter R, Li S-J, Lin C-P, Lowe MJ, Mackay C, Madden DJ, Madsen KH, Margulies DS, Mayberg HS, McMahon K, Monk CS, Mostofsky SH, Nagel BJ, Pekar JJ, Peltier SJ, Petersen SE, Riedl V, Rombouts SARB, Rypma B, Schlaggar BL, Schmidt S, Seidler RD, Siegle GJ, Sorg C, Teng G-J, Veijola J, Villringer A, Walter M, Wang L, Weng X-C, Whitfield-Gabrieli S, Williamson P, Windischberger C, Zang Y-F, Zhang H-Y, Castellanos FX, Milham MP, 2010. Toward discovery science of human brain function. Proc. Natl. Acad. Sci. U. S. A. 107: 4734-9. 
407 Bullmore E, Sporns O, 2012. The economy of brain network organization. Nat. Rev. Neurosci.

$408 \quad 13: 336-349$.

409 Butti C, Sherwood CC, Hakeem AY, Allman JM, Hof PR, 2009. Total number and volume of Von

410 Economo neurons in the cerebral cortex of cetaceans. J. Comp. Neurol. 515: 243-59.

411 Calhoun VD, Adali T, Pearlson GD, Pekar JJ, 2001. A method for making group inferences from

412

413 functional MRI data using independent component analysis. Hum. Brain Mapp. 14: 140-

414

Chai XJ, Castañón AN, Ongür D, Whitfield-Gabrieli S, 2012. Anticorrelations in resting state 415 networks without global signal regression. Neuroimage 59: 1420-8.

416 Chai XJ, Ofen N, Gabrieli JDE, Whitfield-Gabrieli S, 2014. Selective development of 417 anticorrelated networks in the intrinsic functional organization of the human brain. $J$. Cogn. Neurosci. 26: 501-13.

419 Chang C, Glover GH, 2010. Time-frequency dynamics of resting-state brain connectivity 420 measured with fMRI. Neuroimage 50: 81-98.

421 Chumbley JR, Friston KJ, 2009. False discovery rate revisited: FDR and topological inference $422 \quad$ using Gaussian random fields. Neuroimage 44: 62-70.

423 Cole DM, Smith SM, Beckmann CF, 2010. Advances and pitfalls in the analysis and 424 interpretation of resting-state FMRI data. Front. Syst. Neurosci. 4: 8.

425 Cole MW, Reynolds JR, Power JD, Repovs G, Anticevic A, Braver TS, 2013. Multi-task 426 connectivity reveals flexible hubs for adaptive task control. Nat. Neurosci. 16: 1348-55.

427 Cordes D, Haughton VM, Arfanakis K, Wendt GJ, Turski PA, Moritz CH, Quigley MA, 428 Meyerand ME, 2000. Mapping functionally related regions of brain with functional connectivity MR imaging. AJNR. Am. J. Neuroradiol. 21: 1636-44. 
430

431

432

433

434

435

436

437

438

439

440

441

442

443

444

445

446

447

448

449

450

451

452

453

Deshpande G, Santhanam P, Hu X, 2011. Instantaneous and causal connectivity in resting state brain networks derived from functional MRI data. Neuroimage 54: 1043-52.

Di X, Biswal BB, 2013a. Modulatory interactions of resting-state brain functional connectivity. PLoS One 8: e71163.

Di X, Biswal BB, 2013b. Dynamic brain functional connectivity modulated by resting-state networks. Brain Struct. Funct. doi: 10.1007/s00429-013-0634-3.

Di X, Biswal BB, 2014. Identifying the default mode network structure using dynamic causal modeling on resting-state functional magnetic resonance imaging. Neuroimage 86: 53-9.

Dosenbach NUF, Fair DA, Miezin FM, Cohen AL, Wenger KK, Dosenbach RAT, Fox MD, Snyder AZ, Vincent JL, Raichle ME, Schlaggar BL, Petersen SE, 2007. Distinct brain networks for adaptive and stable task control in humans. Proc. Natl. Acad. Sci. U. S. A. 104: $11073-8$.

Doucet G, Naveau M, Petit L, Delcroix N, Zago L, Crivello F, Jobard G, Tzourio-Mazoyer N, Mazoyer B, Mellet E, Joliot M, 2011. Brain activity at rest: a multiscale hierarchical functional organization. J. Neurophysiol. 105: 2753-63.

Fan J, McCandliss BD, Fossella J, Flombaum JI, Posner MI, 2005. The activation of attentional networks. Neuroimage 26: 471-9.

Fischer J, Whitney D, 2012. Attention gates visual coding in the human pulvinar. Nat. Commun. 3: 1051 .

Fox MD, Snyder AZ, Vincent JL, Corbetta M, Van Essen DC, Raichle ME, 2005. The human brain is intrinsically organized into dynamic, anticorrelated functional networks. Proc. Natl. Acad. Sci. U. S. A. 102: 9673-8.

Fox MD, Zhang D, Snyder AZ, Raichle ME, 2009. The global signal and observed anticorrelated resting state brain networks. J. Neurophysiol. 101: 3270-83. 
454 Friston KJ, Buechel C, Fink GR, Morris J, Rolls E, Dolan RJ, 1997. Psychophysiological and modulatory interactions in neuroimaging. Neuroimage 6: 218-29.

456 Friston KJ, Harrison L, Penny W, 2003. Dynamic causal modelling. Neuroimage 19: 1273-302.

457 Gitelman DR, Penny WD, Ashburner J, Friston KJ, 2003. Modeling regional and

458 psychophysiologic interactions in fMRI: the importance of hemodynamic deconvolution.

$459 \quad$ Neuroimage 19: 200-7.

460 Golland Y, Golland P, Bentin S, Malach R, 2008. Data-driven clustering reveals a fundamental

461 subdivision of the human cortex into two global systems. Neuropsychologia 46: 540-53.

462 Greicius MD, Krasnow B, Reiss AL, Menon V, 2003. Functional connectivity in the resting 463 brain: a network analysis of the default mode hypothesis. Proc. Natl. Acad. Sci. U. S. A. 100: $253-258$.

465

466

467

468

469

470

471

472

473

474

475

476
Haynes J-D, Deichmann R, Rees G, 2005. Eye-specific effects of binocular rivalry in the human lateral geniculate nucleus. Nature 438: 496-9.

Kang J, Wang L, Yan C, Wang J, Liang X, He Y, 2011. Characterizing dynamic functional connectivity in the resting brain using variable parameter regression and Kalman filtering approaches. Neuroimage 56: 1222-34.

Keller CJ, Bickel S, Honey CJ, Groppe DM, Entz L, Craddock RC, Lado FA, Kelly C, Milham M, Mehta AD, 2013. Neurophysiological investigation of spontaneous correlated and anticorrelated fluctuations of the BOLD signal. J. Neurosci. 33: 6333-42.

Kelly AMC, Uddin LQ, Biswal BB, Castellanos FX, Milham MP, 2008. Competition between functional brain networks mediates behavioral variability. Neuroimage 39: 527-37.

Von Kriegstein K, Giraud A-L, 2006. Implicit multisensory associations influence voice recognition. PLoS Biol. 4: e326. 
477 Lancaster JL, Tordesillas-Gutiérrez D, Martinez M, Salinas F, Evans A, Zilles K, Mazziotta JC, 478 Fox PT, 2007. Bias between MNI and Talairach coordinates analyzed using the ICBM479152 brain template. Hum. Brain Mapp. 28: 1194-1205.

480 Lancaster JL, Woldorff MG, Parsons LM, Liotti M, Freitas CS, Rainey L, Kochunov P V, 481 Nickerson D, Mikiten SA, Fox PT, 2000. Automated Talairach atlas labels for functional 482 brain mapping. Hum. Brain Mapp. 10: 120-131.

483 Leh SE, Ptito A, Chakravarty MM, Strafella AP, 2007. Fronto-striatal connections in the human 484 brain: A probabilistic diffusion tractography study. 419(2):113-8.

485 Lehéricy S, Ducros M, Van de Moortele P-F, Francois C, Thivard L, Poupon C, Swindale N, 486 Ugurbil K, Kim D-S, 2004. Diffusion tensor fiber tracking shows distinct corticostriatal 487 circuits in humans. Ann. Neurol. 55: 522-9.

488 Liao W, Mantini D, Zhang Z, Pan Z, Ding J, Gong Q, Yang Y, Chen H, 2010. Evaluating the 489 effective connectivity of resting state networks using conditional Granger causality. Biol. 490 Cybern. 102: 57-69.

491 Manoliu A, Riedl V, Doll A, Bäuml JG, Mühlau M, Schwerthöffer D, Scherr M, Zimmer C, Förstl

492 H, Bäuml J, Wohlschläger AM, Koch K, Sorg C, 2013. Insular Dysfunction Reflects

$493 \quad$ Altered Between-Network Connectivity and Severity of Negative Symptoms in

494 Schizophrenia during Psychotic Remission. Front. Hum. Neurosci. 7.

495 Manoliu A, Riedl V, Zherdin A, Mühlau M, Schwerthöffer D, Scherr M, Peters H, Zimmer C, 496 Förstl H, Bäuml J, Wohlschläger AM, Sorg C, 2014. Aberrant Dependence of Default 497 Mode/Central Executive Network Interactions on Anterior Insular Salience Network 498 Activity in Schizophrenia. Schizophr. Bull. 40(2):428-37. 
499 Marrelec G, Krainik A, Duffau H, Pélégrini-Issac M, Lehéricy S, Doyon J, Benali H, 2006.

500 Partial correlation for functional brain interactivity investigation in functional MRI.

$501 \quad$ Neuroimage 32: 228-37.

502 Di Martino a, Scheres a, Margulies DS, Kelly a MC, Uddin LQ, Shehzad Z, Biswal B, Walters

503 JR, Castellanos FX, Milham MP, 2008. Functional connectivity of human striatum: a

504 resting state FMRI study. Cereb. Cortex 18: 2735-47.

505 McAlonan K, Brown VJ, Bowman EM, 2000. Thalamic reticular nucleus activation reflects

506 attentional gating during classical conditioning. J. Neurosci. 20: 8897-901.

507 McAlonan K, Cavanaugh J, Wurtz RH, 2008. Guarding the gateway to cortex with attention in

$508 \quad$ visual thalamus. Nature 456: 391-4.

509 Menon V, Uddin LQ, 2010. Saliency, switching, attention and control: a network model of insula

$510 \quad$ function. Brain Struct. Funct. 214: 655-67.

511 Menon V, 2011. Large-scale brain networks and psychopathology: a unifying triple network

$512 \quad$ model. Trends Cogn. Sci. 15: 483-506.

513 Meunier D, Achard S, Morcom A, Bullmore E, 2009. Age-related changes in modular

514 organization of human brain functional networks. Neuroimage 44: 715-23.

515 Murphy K, Birn RM, Handwerker DA, Jones TB, Bandettini PA, 2009. The impact of global

516 signal regression on resting state correlations: are anti-correlated networks introduced?

$517 \quad$ Neuroimage 44: 893-905.

518 O'Connor DH, Fukui MM, Pinsk MA, Kastner S, 2002. Attention modulates responses in the

519 human lateral geniculate nucleus. Nat. Neurosci. 5: 1203-9.

520 Den Ouden HEM, Daunizeau J, Roiser J, Friston KJ, Stephan KE, 2010. Striatal prediction error

521 modulates cortical coupling. J. Neurosci. 30: 3210-9. 
522 Saad ZS, Gotts SJ, Murphy K, Chen G, Jo HJ, Martin A, Cox RW, 2012. Trouble at rest: how

523 correlation patterns and group differences become distorted after global signal regression.

$524 \quad$ Brain Connect. 2: 25-32.

525 Salvador R, Suckling J, Coleman MR, Pickard JD, Menon D, Bullmore E, 2005.

526 Neurophysiological architecture of functional magnetic resonance images of human brain.

$527 \quad$ Cereb. Cortex 15: 1332-42.

528 Van Schouwenburg MR, den Ouden HEM, Cools R, 2010. The human basal ganglia modulate

529 frontal-posterior connectivity during attention shifting. J. Neurosci. 30: 9910-8.

530 Seeley WW, Menon V, Schatzberg AF, Keller J, Glover GH, Kenna H, Reiss AL, Greicius MD, 531 2007. Dissociable intrinsic connectivity networks for salience processing and executive $532 \quad$ control. J. Neurosci. 27: 2349-56.

533 Shulman GL, Corbetta M, Buckner RL, Fiez JA, Miezin FM, Raichle ME, Petersen SE, 1997 a.

534 Common Blood Flow Changes across Visual Tasks: I. Increases in Subcortical Structures 535 and Cerebellum but Not in Nonvisual Cortex. J. Cogn. Neurosci. 9: 624-647.

536 Shulman GL, Fiez JA, Corbetta M, Buckner RL, Miezin FM, Raichle ME, Petersen SE, $1997 \mathrm{~b}$.

537 Common Blood Flow Changes across Visual Tasks: II. Decreases in Cerebral Cortex. $J$. $538 \quad$ Cogn. Neurosci. 9: 648-663.

539 Smith SM, Fox PT, Miller KL, Glahn DC, Fox PM, Mackay CE, Filippini N, Watkins KE, Toro

540 R, Laird AR, Beckmann CF, 2009. Correspondence of the brain's functional architecture 541 during activation and rest. Proc. Natl. Acad. Sci. U. S. A. 106: 13040-5.

542 Spreng RN, Sepulcre J, Turner GR, Stevens WD, Schacter DL, 2013. Intrinsic architecture 543 underlying the relations among the default, dorsal attention, and frontoparietal control 544 networks of the human brain. J. Cogn. Neurosci. 25: 74-86. 
545 Spreng RN, Stevens WD, Chamberlain JP, Gilmore AW, Schacter DL, 2010. Default network

546 activity, coupled with the frontoparietal control network, supports goal-directed cognition.

$547 \quad$ Neuroimage 53: 303-17.

548 Sridharan D, Levitin DJ, Menon V, 2008. A critical role for the right fronto-insular cortex in 549 switching between central-executive and default-mode networks. Proc. Natl. Acad. Sci.

$550 \quad$ U.S. A. 105: 12569-74.

551 Stephan KE, Kasper L, Harrison LM, Daunizeau J, den Ouden HEM, Breakspear M, Friston KJ, 552 2008. Nonlinear dynamic causal models for fMRI. Neuroimage 42: 649-62.

553 Weissenbacher A, Kasess C, Gerstl F, Lanzenberger R, Moser E, Windischberger C, 2009.

554 Correlations and anticorrelations in resting-state functional connectivity MRI: a

555 quantitative comparison of preprocessing strategies. Neuroimage 47: 1408-16.

556 Wen X, Liu Y, Yao L, Ding M, 2013. Top-down regulation of default mode activity in spatial $557 \quad$ visual attention. J. Neurosci. 33: 6444-53.

558 Yan C, He Y, 2011. Driving and driven architectures of directed small-world human brain $559 \quad$ functional networks. PLoS One 6: e23460.

560 Yeo BTT, Krienen FM, Sepulcre J, Sabuncu MR, Lashkari D, Hollinshead M, Roffman JL, 561 Smoller JW, Zöllei L, Polimeni JR, Fischl B, Liu H, Buckner RL, 2011. The organization 562 of the human cerebral cortex estimated by intrinsic functional connectivity. $J$.

$563 \quad$ Neurophysiol. 106: 1125-65. 


\section{Figure 1}

DMN and task positive networks used in the PPI analysis.

These networks were defined by using spatial ICA. The IC maps were $z$ transformed, and thresholded at $z>1.96$. Maps of all 20 ICs can be found in supplementary Figure S1.
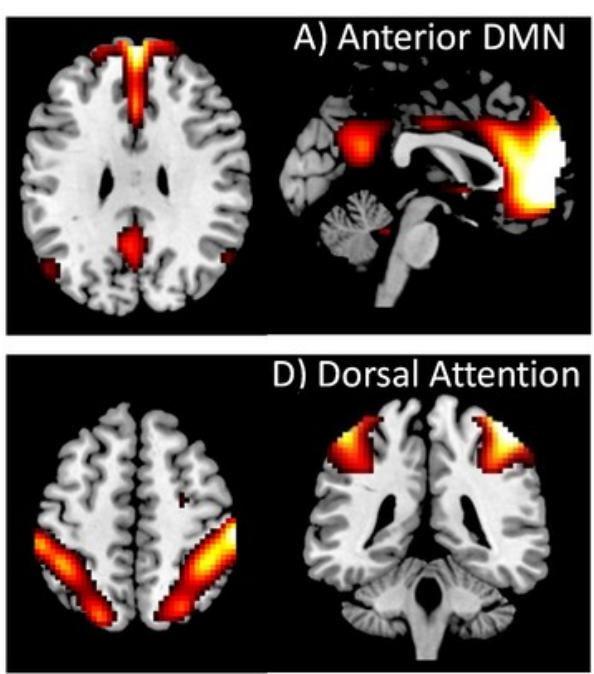
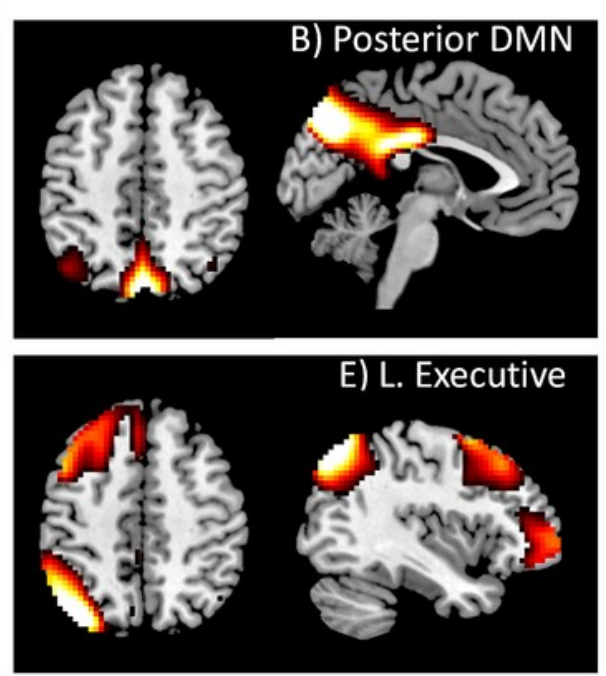
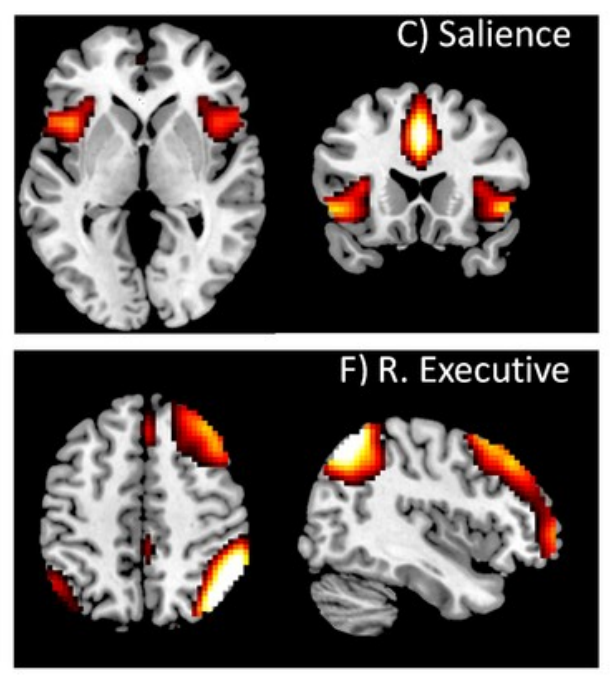


\section{Figure 2}

Results of the network-wise PPI analysis.

Tables indicate the PPI effects between network pairs (row vs. column ). Cells outside the tables represent the dependent variables of the time series of different networks (A-F).

Colored arrows and cells indicate significant PPI effects of a given network (outside cell) and the interaction of two ROls (cells in the tables). Red represents positive effects, while blue represents negative effects. Cells in light gray indicate effects tested but not significant. Statistical significance was determined as $p<0.05$ after Bonferroni correction of all 60 effects tested.
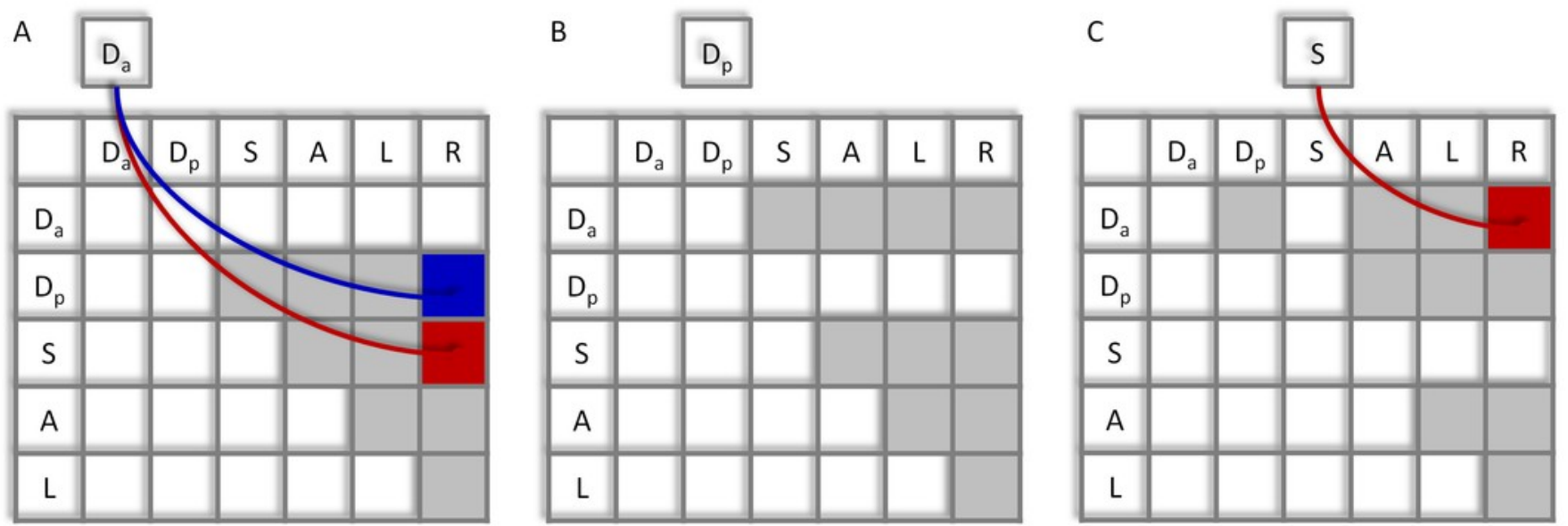

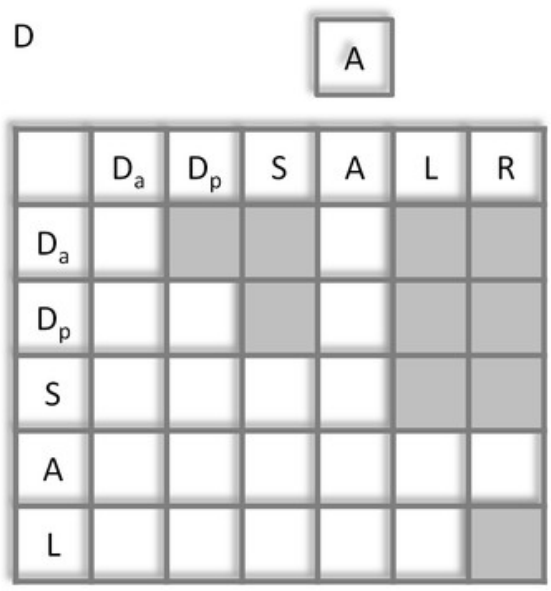

$D_{a}$ : Anterior Default Mode Network A: Dorsal Attention Network

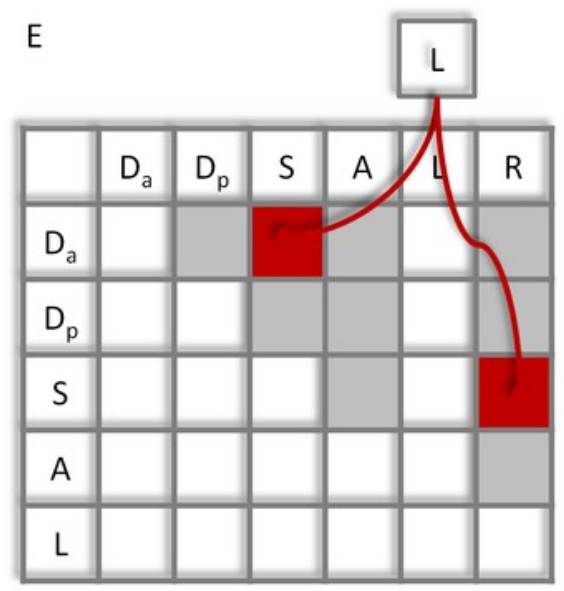

$\mathrm{D}_{\mathrm{p}}$ : Posterior Default Mode Network L: Left Executive Network

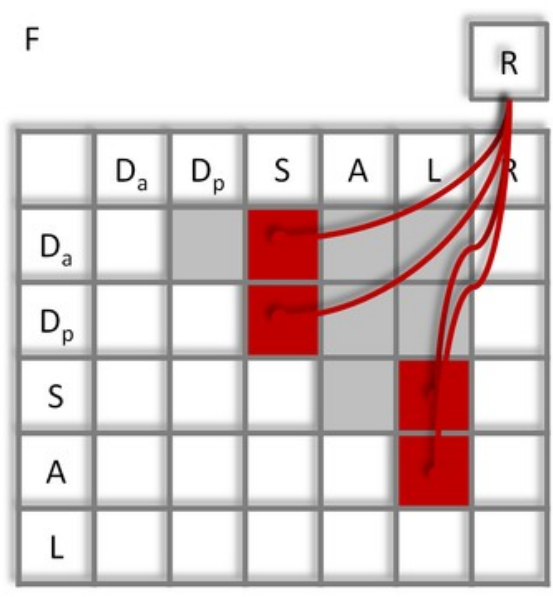

S: Salience Network

R: Right Executive Network 


\section{Figure 3}

Voxel-wise PPI results between the anterior DMN and task positive networks.

Clusters were thresholded at $p<0.001$ with a cluster level FDR correction at $p<0.0033$, which has taken into account of totally 15 voxel-wise analyses. Hot color encodes positive effects, while winter color encodes negative effects. $x$ and $z$ represent $x$ and $z$ coordinates in MNI space.

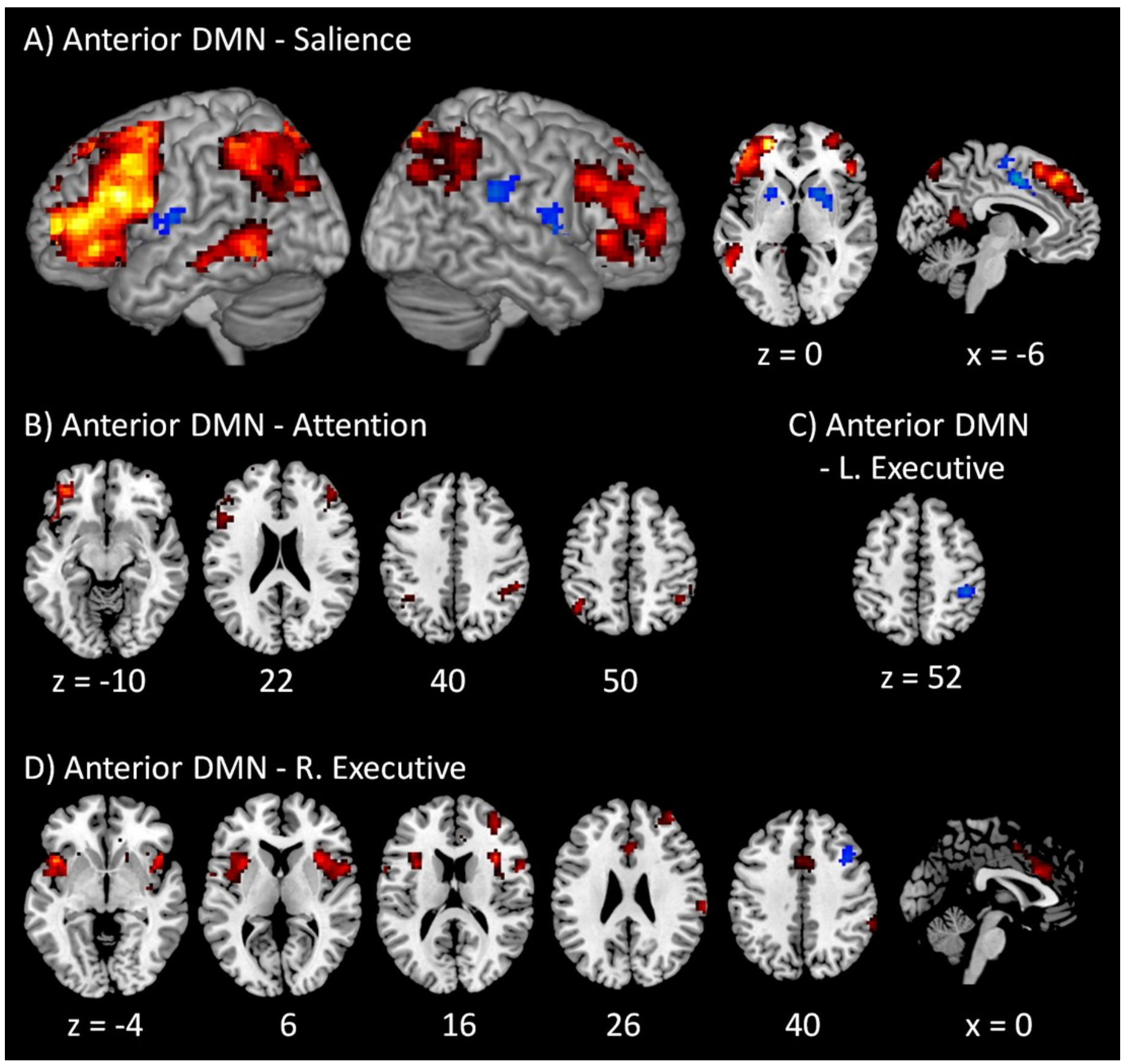




\section{Figure 4}

Voxel-wise PPI results between the posterior DMN and task positive networks.

Clusters were thresholded at $\mathrm{p}<0.001$ with a cluster level FDR correction at $\mathrm{p}<0.0033$, which has taken into account of totally 15 voxel-wise analyses. Hot color encodes positive effects, while winter color encodes negative effects. $x$ and $z$ represents $x$ and $z$ coordinates in MNI space.

\section{A) Posterior DMN - Salience}
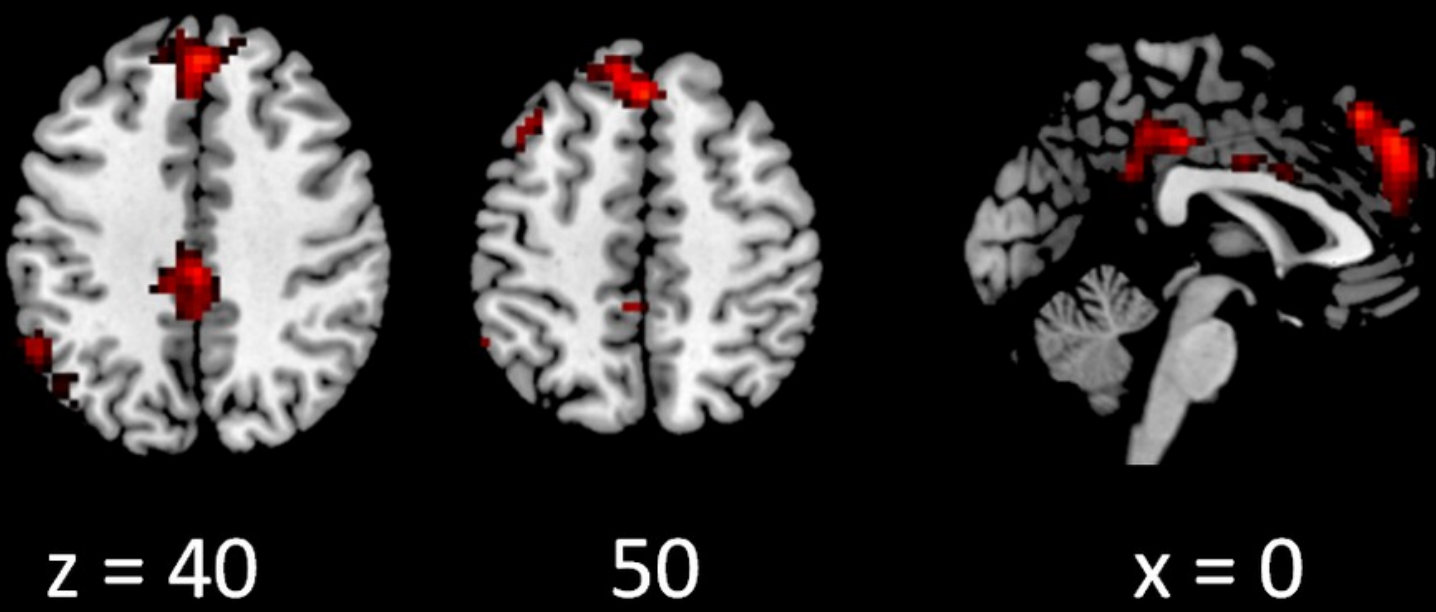

$x=0$

\section{B) Posterior DMN - Attention}
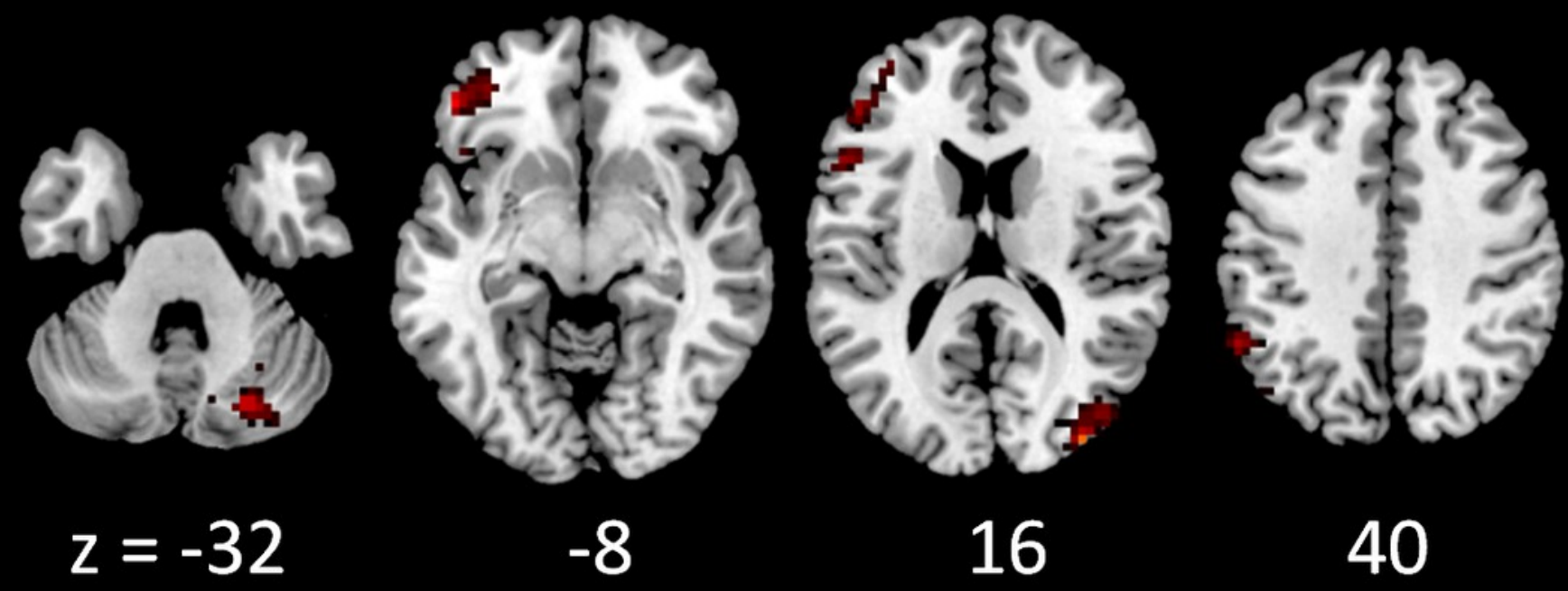

16

40 


\section{Figure 5}

Voxel-wise PPI results between networks within the DMN and within task positive networks.

Clusters were thresholded at $p<0.001$ with a cluster level FDR correction at $p<0.0033$, which has taken into account of totally 15 voxel-wise analyses. Hot color encodes positive effects, while winter color encodes negative effects. $x$ and $z$ represents $x$ and $z$ coordinates in MNI space. 


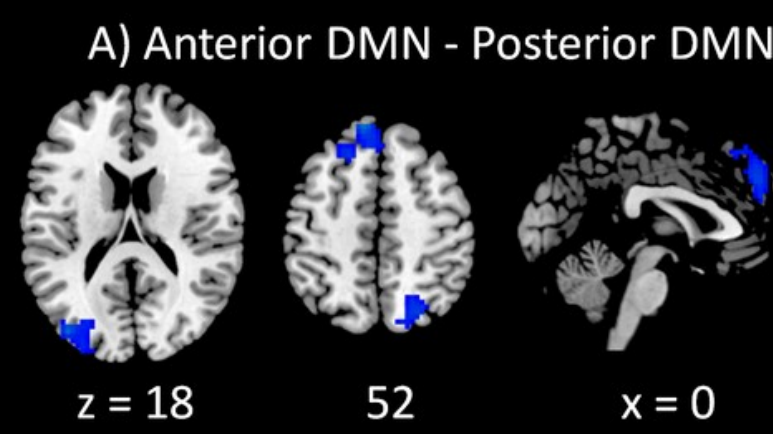

B) Salience - Attention

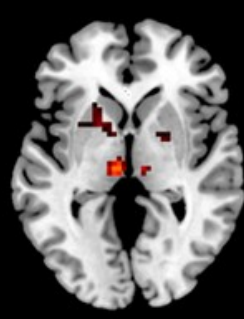

$\mathrm{z}=0$

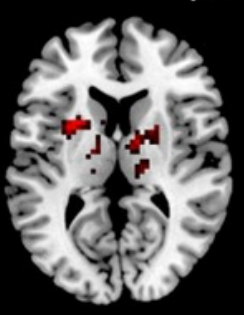

10

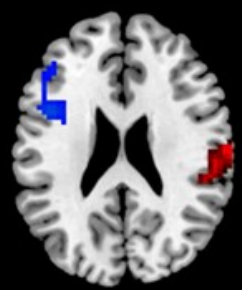

24

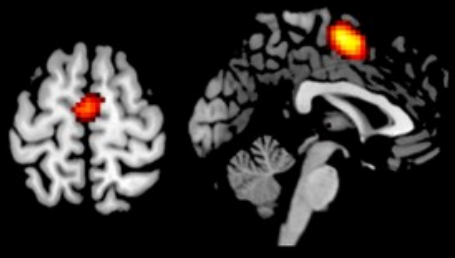

64$$
x=0
$$

C) Salience - L. Executive

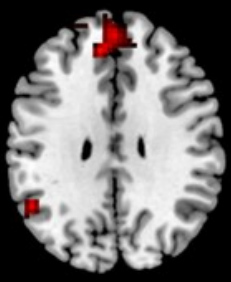

$z=31$

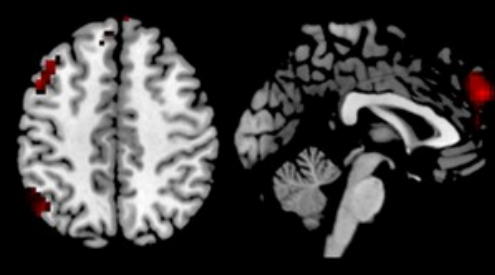

46

$x=0$

D) Salience - R. Executive

E) Attention - L. Executive

F) Attention - R. Executive

G) L. Executive - R. Executive

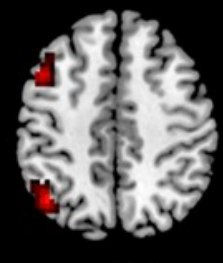

$z=46$
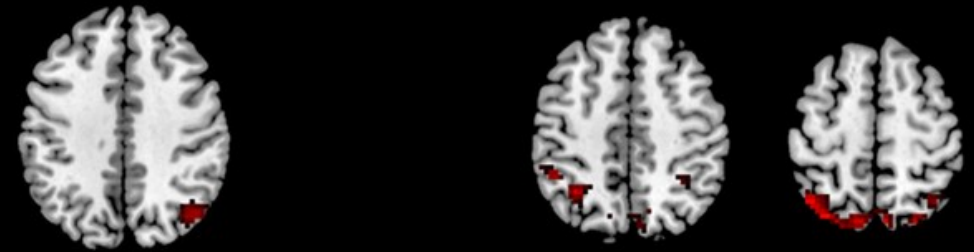

$z=40$

$z=48$

58 


\section{Table 1 (on next page)}

Mean correlations (Fisher's z scores) among the six networks.

Values in brackets represent raw $p$ values of corresponding cross subject one sample t-test. Bold font indicates statistically significant after Bonferroni multiple comparison correction of totally 15 correlations. 
Table 1 Mean correlations (Fisher's z scores) among the six networks.

Values in brackets represent raw $\mathrm{p}$ values of corresponding cross subject one sample t-test. Bold font indicates statistically significant after Bonferroni multiple comparison correction of totally 15 correlations.

\begin{tabular}{|c|c|c|c|c|c|}
\hline & $\begin{array}{l}\text { Anterior } \\
\text { DMN }\end{array}$ & $\begin{array}{l}\text { Posterior } \\
\text { DMN }\end{array}$ & Salience & Dorsal Attention & L. Executive \\
\hline Posterior DMN & $\begin{array}{c}0.359 \\
\left(7.01 \times 10^{-23}\right)\end{array}$ & & & & \\
\hline Salience & $\begin{array}{c}-0.299 \\
\left(1.34 \times 10^{-16}\right)\end{array}$ & $\begin{array}{c}-0.251 \\
\left(4.75 \times 10^{-15}\right)\end{array}$ & & & \\
\hline Dorsal Attention & $\begin{array}{c}-0.530 \\
\left(1.55 \times 10^{-28}\right)\end{array}$ & $\begin{array}{c}-0.055 \\
(0.0051)\end{array}$ & $\begin{array}{c}0.333 \\
\left(8.45 \times 10^{-16}\right)\end{array}$ & & \\
\hline L. Executive & $\begin{array}{c}0.184 \\
\left(8.25 \times 10^{-10}\right)\end{array}$ & $\begin{array}{c}0.320 \\
\left(1.05 \times 10^{-22}\right)\end{array}$ & $\begin{array}{c}0.076 \\
(0.0041)\end{array}$ & $\begin{array}{l}0.003 \\
(0.87)\end{array}$ & \\
\hline R. Executive & $\begin{array}{c}0.247 \\
\left(2.37 \times 10^{-13}\right)\end{array}$ & $\begin{array}{c}0.188 \\
\left(3.09 \times 10^{-12}\right)\end{array}$ & $\begin{array}{c}-0.142 \\
\left(1.09 \times 10^{-7}\right)\end{array}$ & $\begin{array}{l}0.004 \\
(0.87)\end{array}$ & $\begin{array}{c}0.427 \\
\left(3.83 \times 10^{-28}\right)\end{array}$ \\
\hline
\end{tabular}

L., left.

R., right. 